\title{
Importance of repulsive interactions for the equation of state and other properties of strongly interacting matter 1
}

\author{
V. V. Begun ${ }^{a, b}$ \\ ${ }^{a}$ Jan Kochanowski University, Kielce, Poland \\ ${ }^{b}$ Bogolyubov Institute for Theoretical Physics, Kiev, Ukraine
}

\begin{abstract}
We illustrate the role of repulsive interactions in a hadron-resonance gas at freeze-out and in a gas of quark-gluon bags. Taking into account non-zero size of particles in hadron gas leads to a significant decrease and shift of the net-baryon density maximum. The transition point from baryon to meson dominated matter depends on the difference between baryon and meson radii. We also show that depending on the properties of the quark-gluon bags one may obtain any type of the phase transition from hadron gas to quark-gluon plasma: the first or second order, as well as four types of the crossover.
\end{abstract}

\section{Introduction}

The hadron-resonance gas (HRG) model has become a standard tool for the analysis of heavy ion collisions. With only a few parameters the HRG model allows us to describe numerous ratios of particle multiplicities produced in the wide energy range from SIS, the AGS and SPS to RHIC and the LHC [1, 2, 3, 4]. The proper definition of the space-time geometry and hydrodynamic flow at freeze-out allows to further extend the HRG model to describe the transverse-momentum spectra and other soft-hadronic observables [5, 6, 7]. Resonances introduced to the ideal HRG effectively take into account attractive interactions. However, the nucleon-nucleon potential includes both attraction at large distances and repulsion at small distances. Repulsive interactions can be included in HRG using the van der Waals excluded volume of particles [8]. One should keep in mind that point-like hadrons would always become dominant phase at very high energy density due to the large number of different types of hadrons. Only the presence

${ }^{1}$ Talk at the 32th Max-Born-Symposium and HECOLS workshop on "Three Days of Phase Transitions in Compact Stars, Heavy-Ion Collisions and Supernovae", Institute for Theoretical Physics, University of Wrocław, Wrocław, Poland, February 17-19, 2014. 
of the excluded volume effects ensures a phase transition from the gas of hadrons and resonances to the quark-gluon plasma (QGP) [9].

\section{Gas of extended hadrons and resonances}

In Ref. [10] we calculated the excluded volume effects along the chemical freeze-out line obtained for the central $\mathrm{Pb}+\mathrm{Pb}(\mathrm{Au}+\mathrm{Au})$ collisions registered by experiments at SIS, the AGS, SPS, and RHIC [1, 2]. If all particles have the same volume, then the Boltzmann approximation gives the same suppression factor for all densities, which cancels in the particle ratios. However, the absolute value, for example, of the net-baryon density strongly depends on the proper volume and collision energy.

A moderate estimate of the particle radius by $r=0.5 \mathrm{fm}$ leads to the suppression factor of about 0.75 at the AGS and 0.5 at the SPS, see [10]. This non-monotonous suppression with energy shifts the maximum of the net-baryon density to lower energies, see the left panel of Fig. 1. For the proper radii equal to 0.5 and $1 \mathrm{fm}$ the density maximum moves from 34 to 17 and $7 \mathrm{AGeV}$, correspondingly.

The transition point from baryon to meson dominated matter is sensitive only to the difference between baryon and meson radii. It happens because at freeze-out the Boltzmann approximation works well for entropy densities. Then, for equal baryon and meson radii the corresponding entropies are suppressed by the same factor and cross at the same point. For the baryon radii $r_{B}=0.5 \mathrm{fm}$ and meson radii $r_{M}=0$, the baryon/meson transition point moves from 46 to $23 \mathrm{AGeV}$, see the right panel of Fig. 1 .

\section{Cluster Plasma}

One may extend the HRG model by adding the spectrum of bags filled with non-interacting massless quarks and gluons to the usual HRG spectrum. This is reasonable at high temperatures and densities, where individual hadrons melt and merge, forming bigger states with larger masses and volumes. In this way the bags may mimic the effect of heavy resonances which have not been found yet. These resonances/bags are not allowed to overlap, in the same way as usual hadrons with the excluded volume. Interestingly, in such a system one can study the transition from HRG to QGP analytically.

The possibility of phase transitions in the gas of quark-gluon bags was demonstrated for the first time in Ref. [13. Further studies allowed to obtain 

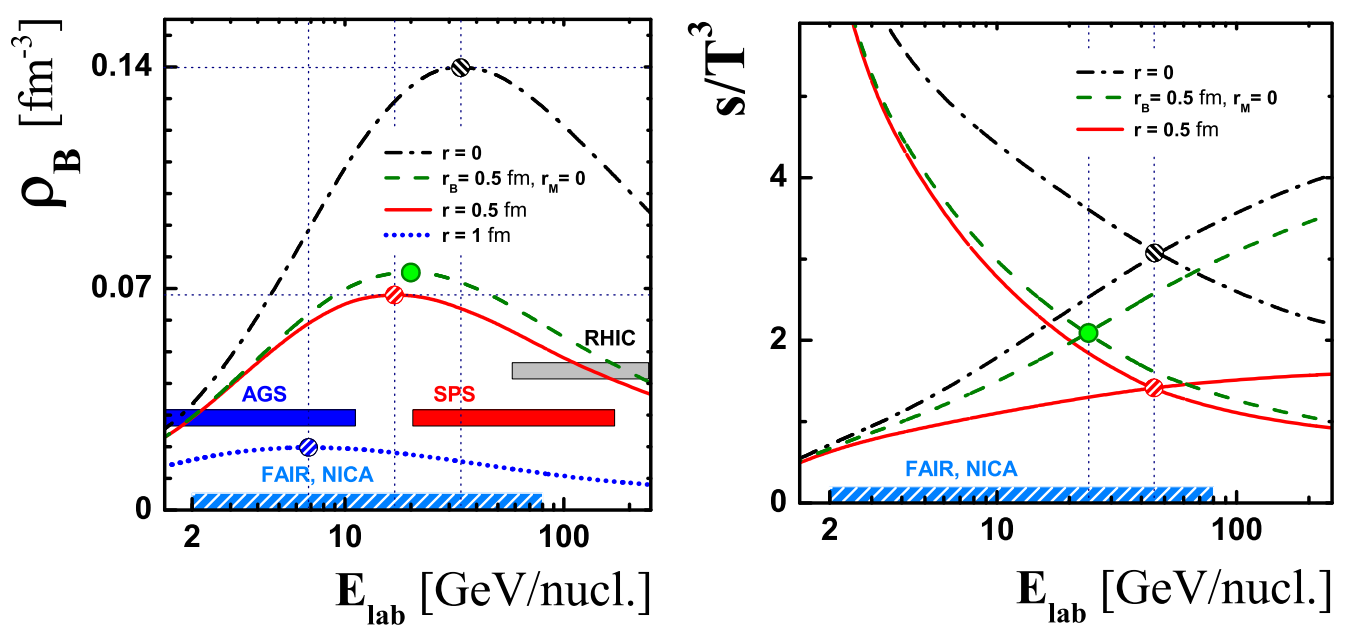

Figure 1: Left: The net-baryon density along the chemical freeze-out line [1, 2. Dashed-dotted line corresponds to the model of point-like particles with $r=0$, dashed line to $r_{B}=0.5 \mathrm{fm}$ and $r_{M}=0$, solid line to $r_{B}=r_{M}=r=$ $0.5 \mathrm{fm}$, and dotted line to $r_{B}=r_{M}=r=1 \mathrm{fm}$. Right: The ratios of baryon and meson entropies to the temperature cubed, $s / T^{3}$, along the chemical freeze-out line. At small energies baryons dominate, while at large energies mesons take over. Dashed-dotted lines correspond to the model with $r=0$, solid lines to $r_{B}=r_{M}=r=0.5 \mathrm{fm}$, and dashed lines to $r_{B}=0.5 \mathrm{fm}$ and $r_{M}=0$. The horizontal bands indicate the energy range of previous, current, and future experimental facilities for heavy-ion collisions.

the 1st, 2nd, and higher order transitions [14, 15, 16, 17, 18]. A possibility of no phase transitions was also pointed out in Ref. [14, 15]. It was suggested [19] to model a smooth crossover transition by the gas of quark-gluon bags. One can show that the type of the transition from QGP to HRG is not sensitive to the presence of the HRG, and depends only on the form of the mass-volume spectrum. In Ref. 20] we summarized all known possibilities and found even richer structure for the case of crossover.

The results are illustrated by the behavior of the average bag size with increasing temperature, see Fig. 2. In the case of a phase transition the average bag size increases and occupies the whole system. There are also two similar cases for the case of crossover, see [20] for more details. However, there are two more possibilities. In spite of the same asymptotic behavior for the pressure and energy density, the average bag size may be constant or even decrease to the minimal value in thermodynamic limit. 


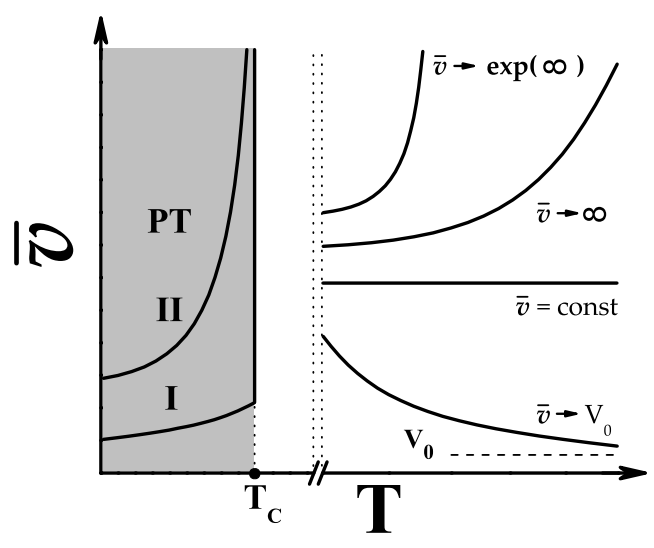

Figure 2: The schematic view of the possible temperature dependence of the average volume of the bag. The PT I and II labels denote the phase transition of the first and the second order, correspondingly. The right part of the figure shows the possible behavior of the bag after the crossover.

\section{Conclusions}

We have shown that the effect of taking into account the non-zero proper volume of particles can be very strong. It leads to smaller particle number and entropy densities and moves the position of the net baryon density maximum to lower energies. The transition point from baryon to meson domination is always situated at higher energies than the density maximum. The transition point can move to lower energies only for different meson and baryon radii in the system. A more precise estimate of the baryon density maximum and transition point can be important for the search on the compressed baryonic matter at GSI [11, 12].

The extended HRG model with quark-gluon bags can give any type of the transition between HRG and QGP depending on the bag properties. One may even get a cluster QGP, which is different from the ideal QGP, despite the similar equation of state.

\section{Acknowledgment}

This work was supported by Polish National Science Center grant No. DEC-2012/06/A/ST2/00390. 


\section{References}

[1] F. Becattini, J. Manninen and M. Gazdzicki, Phys. Rev. C 73, 044905 (2006).

[2] J. Cleymans, H. Oeschler, K. Redlich and S. Wheaton, Phys. Rev. C 73, 034905 (2006).

[3] M. Petran, J. Letessier, V. Petracek and J. Rafelski, Phys. Rev. C 88, 034907 (2013).

[4] J. Stachel, A. Andronic, P. Braun-Munzinger and K. Redlich, J. Phys. Conf. Ser. 509, 012019 (2014).

[5] W. Broniowski and W. Florkowski, Phys. Rev. Lett. 87, 272302 (2001).

[6] W. Broniowski, A. Baran and W. Florkowski, AIP Conf. Proc. 660, 185 (2003).

[7] V. Begun, W. Florkowski and M. Rybczynski, arXiv:1405.7252 [hep-ph].

[8] M. I. Gorenstein, V. K. Petrov and G. M. Zinovev, Phys. Lett. B 106, 327 (1981).

[9] J. Cleymans, M. I. Gorenstein, J. Stalnacke and E. Suhonen, Phys. Scripta 48, 277 (1993).

[10] V. V. Begun, M. Gazdzicki and M. I. Gorenstein, Phys. Rev. C 88, no. 2, 024902 (2013).

[11] B. Friman, C. Hohne, J. Knoll, S. Leupold, J. Randrup, R. Rapp and P. Senger, Lect. Notes Phys. 814, 1 (2011).

[12] T. Galatyuk, Investigation of baryon rich dense nuclear matter at SIS100, "CPOD-2013", March 11-15, 2013, Napa, California, USA [https://www-alt.gsi.de/documents/DOC-2013-Mar-43-1.pdf].

[13] M. I. Gorenstein, V. K. Petrov, and G. M. Zinovjev, Phys. Lett. B 106 (1981) 327;

[14] M. I. Gorenstein, W. Greiner, and Shin Nan Yang, J. Phys. G 24 (1998) 725 . 
[15] M. I. Gorenstein, M. Gazdzicki, and W. Greiner, Phys. Rev. C 72 (2005) 024909 .

[16] I. Zakout, C. Greiner, and J. Schaffner-Bielich, Nucl. Phys. A 781 (2007) 150.

[17] K. A. Bugaev, Phys. Rev. C 76 (2007) 014903;

[18] A. Bessa, E. S. Fraga, and B. W. Mintz, Phys. Rev. D 79 (2009) 034012.

[19] L. Ferroni and V. Koch, Phys. Rev. C 79 (2009) 034905.

[20] V. V. Begun, M. I. Gorenstein and W. Greiner, J. Phys. G 36, 095005 (2009). 